\title{
Alterações da autoestima em pacientes oncológicos submetidos ao tratamento quimioterápico
}

\author{
Changes in self-esteem in oncological patients undergoing chemotherapy
}

Cambios en la autoestima en pacientes oncológicos sometidos a la quimioterapia

Francisco Braz Milanez Oliveira ${ }^{1}$, Bárbara Mônica Lopes e Silva ${ }^{1}$, Bianca Santos Soares ${ }^{1}$, Brunna Matos Sousa ${ }^{1}$, Chrisllayne Oliveira da Silva ${ }^{1 *}$, Francilene Pinho Barbosa ${ }^{1}$, Francimar Ribeiro da Silva ${ }^{1}$, Priscila Pontes Araújo Sousa ${ }^{1}$, Raimundo Nonato dos Santos Filho ${ }^{1}$, Thalyta D'Paula Dantas do Nascimento ${ }^{1}$, Wenderson Costa da Silva ${ }^{1}$, Franklin Roosevelt Oliveira Maranhão ${ }^{2}$, Renan Rhonalty Rocha ${ }^{3}$, Rita Maria Pereira da Paz ${ }^{4}$, Tatyanne Maria Pereira de Oliveira ${ }^{5}$

\section{RESUMO}

Objetivo: Analisar as produções científicas sobre a avaliação da autoestima de pacientes oncológicos, submetidos ao tratamento quimioterápico. Metodologia: Trata-se de uma revisão integrativa da literatura, onde foi formulada a seguinte questão PICo (não-clínica): Quais as evidências cientificas apontam as mudanças na autoestima dos pacientes oncológicos submetidos ao tratamento quimioterápico? Resultados: As alterações na autoestima em pacientes oncológicos estão relacionadas a efeitos adversos da quimioterapia, como a alopecia, e ao estado psicológico baixo associado à depressão, comprometimento familiar, isolamento social, estresse, e alguns sintomas como náuseas, vômitos, anorexia, debilidade física, que provocam sentimento de impotência e desesperança afetando diretamente na autoestima. A produção dos bundles de intervenção propôs estimular a pratica de atividade física e outras atividades de lazer em pacientes oncológicos, oferecer um programa de apoio psicossocial integrado para os pacientes e seus acompanhantes no enfrentamento da doença e mudança na autoestima, Orientar sobre o surgimento da alopecia durante a quimioterapia, e os efeitos negativos na autoimagem, Promover atividades de apoio ao retorno ao trabalho e aconselhamento ocupacional em pacientes oncológicos, Desenvolver atividades de embelezamento e do uso de adornos em pacientes submetidos a quimioterapia. Considerações Finais: $O$ tratamento quimioterapico pode ser considerado um fator determinante nas alterações da autoestima, pois possibilita a ocorrência de inúmeros impactos na vida do paciente oncológico, tais coomo, fisíco, emocionais e sociais.

Palavras-chaves: Autoimagem, Pacientes, Neoplasias, Quimioterapia.

\begin{abstract}
Objective: To analyze the scientific production on the evaluation of the self-esteem of cancer patients, submitted to chemotherapy treatment. Methodology: This is an integrative review of the literature, where the following question was formulated PICo (non-clinical): What scientific evidence points to the changes in the self-esteem of cancer patients submitted to chemotherapy? Results: Changes in self-esteem in cancer patients are related to adverse effects of chemotherapy, such as alopecia, and low psychological state associated with depression, family involvement, social isolation, stress, and some symptoms such as nausea, vomiting, anorexia, physical weakness, which provoke feelings of impotence and hopelessness directly affecting self-esteem. The production of intervention bundles proposed to stimulate the practice of physical activity and other leisure activities in cancer patients, offer an integrated psychosocial support program for

\footnotetext{
${ }^{1}$ Fac. de Ciências e Tecnologia do Maranhão (FACEMA). Caxias-MA. * E-mail: chris-layne10@hotmail.com

2 Coordenador do SAMU Regional Caxias-MA.

${ }_{3}^{3}$ Mestrando em Biotecnologia pela Universidade Federal do Ceará-UFC.

${ }^{4}$ Especialista em Docência do Ensino Superior em Enfermagem.

${ }^{5}$ Mestranda em Ciências e Saúde pela Universidade Federal do Piauí-UFPI.
} 
patients and their companions in coping with the disease and change in self-esteem, Guidance on the emergence of alopecia during chemotherapy, and negative effects on self-image, Promote activities to support return to work and occupational counseling in cancer patients, Develop activities for embellishment and use of adornments in patients undergoing chemotherapy. Final Considerations: Chemotherapeutic treatment can be considered a a determinant factor in the alterations of the self-esteem, because it allows the occurrence of innumerable impacts on the life of the oncological patient, such as, physical, emotional and social.

Keywords: Self-esteem, Patients, Neoplasms, Chemotherapy.

\section{RESUMEN}

Objetivo: Analizar las producciones científicas sobre la evaluación de la autoestima de pacientes oncológicos, sometidos al tratamiento quimioterápico. Metodología: Se trata de una revisión integrativa de la literatura, donde se formuló la siguiente pregunta PICo (no clínica): ¿Cuáles son las evidencias científicas que apuntan a los cambios en la autoestima de los pacientes oncológicos sometidos al tratamiento quimioterápico? Resultados: Las alteraciones en la autoestima en pacientes oncológicos están relacionadas a efectos adversos de la quimioterapia, como la alopecia, y al estado psicológico bajo asociado a la depresión, compromiso familiar, aislamiento social, estrés, y algunos síntomas como náuseas, vómitos, anorexia, debilidad física, que provocan sentimientos de impotencia y desesperanza afectando directamente a la autoestima. La producción de los lotes de intervención propuso estimular la práctica de actividad física y otras actividades de ocio en pacientes oncológicos, ofrecer un programa de apoyo psicosocial integrado para los pacientes y sus acompañantes en el enfrentamiento de la enfermedad y cambio en la autoestima, Orientar sobre el surgimiento de la alopecia durante la quimioterapia, y los efectos negativos en la autoimagen, Promover actividades de apoyo al retorno al trabajo y asesoramiento ocupacional en pacientes oncológicos, Desarrollar actividades de embellecimiento y del uso de adornos en pacientes sometidos a quimioterapia. Consideraciones Finales: El tratamiento quimioterapico puede ser considerado un " un factor determinante en las alteraciones de la autoestima, pues posibilita la ocurrencia de innumerables impactos en la vida del paciente oncológico, tales coo, físico, emocional y social.

Palabras claves: Autoestima, Pacientes, Neoplasias, Quimioterapia.

\section{INTRODUÇÃO}

As neoplasias representam um grande problema de saúde pública e uma das principais causas de óbitos, pois pode ser ocasionado em qualquer faixa etária, não havendo medidas de prevenção totalmente definidas, mas que contribuem para diminuir os riscos para o câncer. Existem diversos conceitos na literatura oncológica sobre o câncer, que pode ser definido como o crescimento anormal de células que invadem os tecidos do corpo. Se o câncer tiver início em tecidos do epitélio, como pele e mucosas é classificado como carcinoma, se tiver início em tecidos conjuntivos como ossos, músculos ou cartilagem é denominado como sarcoma. Quando o processo ocorre através da disseminação para outras partes do corpo denomina-se metástase (BRASIL, 2009).

É notório que as neoplasias não ocasionam somente alterações na funcionalidade e estruturas corporais mas também afetam o psicológico e emocional do paciente, por se tratar de uma patologia de início silenciosa e com evolução que tende a ser de médio a grave quanto às perdas da funcionalidade orgânica, inclusive em muitos casos podendo levar a óbito (Brasil, 2014).

As condutas terapêuticas mais utilizadas no tratamento oncológico são as cirurgias, radioterapias e a quimioterapia (BRASIL, 2014). A quimioterapia é a forma de tratamento sistêmico do câncer que usa medicamentos denominados "quimioterápicos" (ou antineoplásicos) administrados em intervalos regulares, que variam de acordo com os esquemas terapêuticos (SILVA J, SILVA MJS, 2017). A indústria farmacêutica vem desenvolvendo drogas para tratar o câncer, que tenham efeitos tóxicos menos agressivos, os quais além de bem toleráveis, também são de fácil manejo (MARQUES, PIERIN, 2008). 
A utilização da quimioterapia antineoplásica tem se tornando uma das mais importantes para combater o câncer. É importante destacar que o paciente pode ter seu equilíbrio psicológico ameaçado pelas mudanças que serão necessárias no decorrer da doença e dos tratamentos, incluindo alterações em sua autoestima (SOARES, SILVA, 2010). Além disso, os benefícios dos tratamentos de câncer são, em geral, difíceis de definir, em razão de efeitos colaterais subjetivos que influenciam na qualidade de vida (SCHMIDT, HAHN, 2014).

Nessa perspectiva, surge a necessidade da equipe de enfermagem se sensibilizar para as mudanças ocorridas na autoestima dos pacientes oncológicos. A autoestima pode ser definida como a ausência de afeto positivo que o indivíduo tem de si próprio, sendo de grande relevância na sua relação com o outro (BORDA, RINCÓN; PEREZ, 2012).

É essencial que os profissionais da saúde venham analisar as mudanças na autoestima dos pacientes com câncer submetido a tratamentos como a quimioterapia, para assim prestar uma melhor assistência, bem como avaliar e controlar a eficácia do tratamento (FORONES et al, 2006).

Não obstante surge a necessidade da equipe de enfermagem de se sensibilizar e avaliar as mudanças ocorridas quanto à autoestima de pacientes oncológicos, através de bundles, que consiste em um conjunto de práticas baseadas em evidências com finalidade contribuir para a melhoria nas práticas de cuidado.

Assim foi definida a questão problematizadora desta revisão sendo: Quais as evidências cientificas apontam as mudanças na autoestima dos pacientes oncológicos submetidos ao tratamento quimioterápico? Para tal, o objetivo desta revisão foi analisar as produções científicas sobre a avaliação da autoestima de pacientes oncológicos, submetidos ao tratamento quimioterápico, e elaborar bundles de intervenções para a aplicação na assistência de enfermagem acerca da problemática.

\section{METODOLOGIA}

Trata-se de uma revisão integrativa da literatura, que é considerado um recurso da prática baseada em evidência, que resume uma retrospectiva da literatura empírica ou teórica, proporcionando uma compreensão mais abrangente de um fenômeno em particular e identificar as melhores evidências disponíveis sobre o efeito de determinada terapia ou intervenção, para que dessa maneira os profissionais tenham conhecimento das inovações e melhorias nas práticas descritas em literatura (BROOME, 2006).

Utilizando os níveis de evidência e graus de recomendação, que é uma proposta de organização das informações quanto à confiabilidade, qualidade e validade da informação. Onde os níveis de evidência incluem os tipos de pesquisa sendo numerados de 1-7, sendo o nível mais alto revisão sistemática (nível 1), o nível de evidência está relacionado a qualidade da informação. Enquanto que o grau de recomendação é definido em $A$ (resultados que recomendam a intervenção), B (resultados não conclusivos) e C (não recomenda a intervenção), portanto o grau de recomendação está relacionado a conclusão da pesquisa (BORK, 2011).

Tendo como etapas para sua elaboração: definição do objetivo; estabelecimento de critérios de inclusão e exclusão para a seleção da amostra; definição das informações a serem extraídas dos artigos selecionados; análise, e discussão dos resultados. Para nortear o estudo, foi formulada a seguinte questão PICo (não clínica): Quais as evidências cientificas apontam as mudanças na autoestima dos pacientes oncológicos submetidos ao tratamento quimioterápico?

Quanto a construção da questão que conduziu esta revisão integrativa da literatura. Utilizou-se a estratégia PICo, definindo a inicial (P) para Paciente, (I) para Intervenção, (Co) para o Contexto, aplicando os descritores indexados nos idiomas português, inglês e espanhol. Onde os descritores foram obtidos a partir do Medical Subject Headings (MESH), dos Descritores em Ciências da Saúde (DeCS) e dos Títulos CINAHL. 
A coleta de dados foi realizada através da busca eletrônica no período de maio de 2018 nas seguintes bases de dados: Bireme (Biblioteca Virtual de Saúde - BVS), PubMed da National Library of Medicine e CINAHL(Cumulative Index to Nursing and Allied Health Literature).

Os critérios de inclusão definidos foram: estudos primários, disponíveis em sua totalidade, publicados nos últimos cinco anos, de 2013 até 2018, nos idiomas Português, Espanhol e Inglês. Foram excluídos da busca inicial capítulos de livros, resumos, textos incompletos, teses de doutorado, dissertações de mestrados, monografias e relatos técnicos.

Utilizaram-se os seguintes bancos com seus respectivos descritores:

Quadro 1 - elementos da estratégia PICo e descritores utilizados. Caxias, Ma, 2018.

\begin{tabular}{|c|c|c|c|c|}
\cline { 2 - 5 } \multicolumn{1}{c|}{} & Elementos & Mesh & Decs & Títulos Cinahl \\
\hline P & $\begin{array}{c}\text { "Paciente" } \\
\text { "Câncer" }\end{array}$ & $\begin{array}{c}\text { "Patients" } \\
\text { "Neoplasms" }\end{array}$ & $\begin{array}{c}\text { "Pacientes" } \\
\text { "Neoplasias" }\end{array}$ & "Cancer patient" \\
\hline I & "Autoestima" & "Self concept" & "Autoimagem" & "Self concept" \\
\hline Co & "Quimioterapia" & "Drug therapy" & $\begin{array}{c}\text { "tratamento } \\
\text { farmacológico" }\end{array}$ & "Drug therapy" \\
\hline
\end{tabular}

Fonte: Dados da pesquisa, 2018.

Durante a pesquisa foram utilizados os descritores, e utilizando o operador booleano "And" combinados nos bancos de dados, resultando em estratégias específicas em cada base:

Quadro 2 - Estratégias de busca utilizadas nas bases de dados. Caxias, Ma, 2018.

\begin{tabular}{|c|c|c|c|c|}
\hline Base & Estratégia de busca & Resultados & Filtrados & Selecionados \\
\hline $\begin{array}{c}\text { Bireme } \\
\text { (descritor } \\
\text { es Decs) }\end{array}$ & $\begin{array}{l}\text { tw:((tw:(patient)) AND (tw:(neoplasms)) AND } \\
\text { (tw:(self concept )) AND (tw:(drug therapy))) AND } \\
\text { (instance:"regional") AND ( fulltext:("1") AND } \\
\text { year_cluster:("2013" OR "2016" OR "2014" OR } \\
\text { "2015" OR "2017")) }\end{array}$ & 86 & 21 & 8 \\
\hline $\begin{array}{l}\text { PubMed } \\
\text { (descripto } \\
\text { rs MeSH) }\end{array}$ & 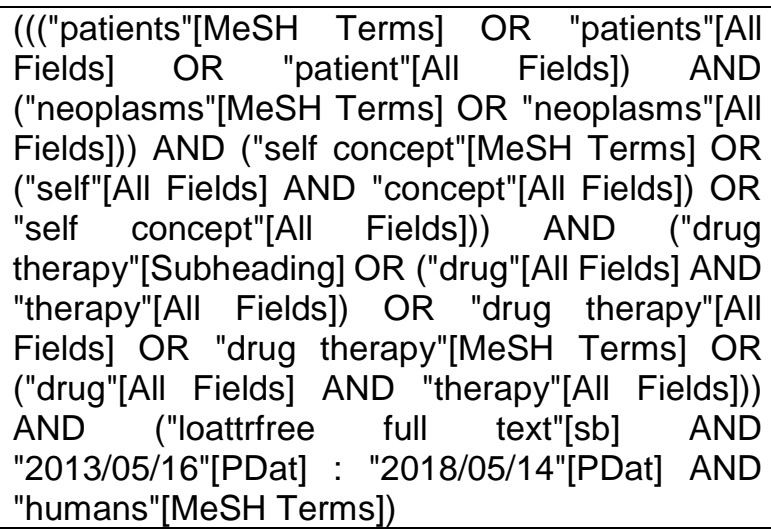 & 345 & 29 & 6 \\
\hline $\begin{array}{c}\text { CINAHL } \\
\text { (CINAHL } \\
\text { Headings) }\end{array}$ & $\begin{array}{l}\text { (cancer patients) AND (self concept) AND (drug } \\
\text { therapy) }\end{array}$ & 10 & 10 & 7 \\
\hline
\end{tabular}

Fonte: Elaboração dos autores. 
Os estudos foram pré-selecionados segundo os critérios de inclusão e exclusão e de acordo com a estratégia de funcionamento e busca de cada base de dados, conforme fluxograma descrito na Figura 1.

Figura 1: Fluxo do processo de seleção dos estudos para a revisão integrativa. Caxias-MA, 2018. (n=8).

Questão norteadora: Quais as evidências cientiificas apontam as alterações de autoestima em pacientes oncologicos submetidos ao tratamento quimiterapico?

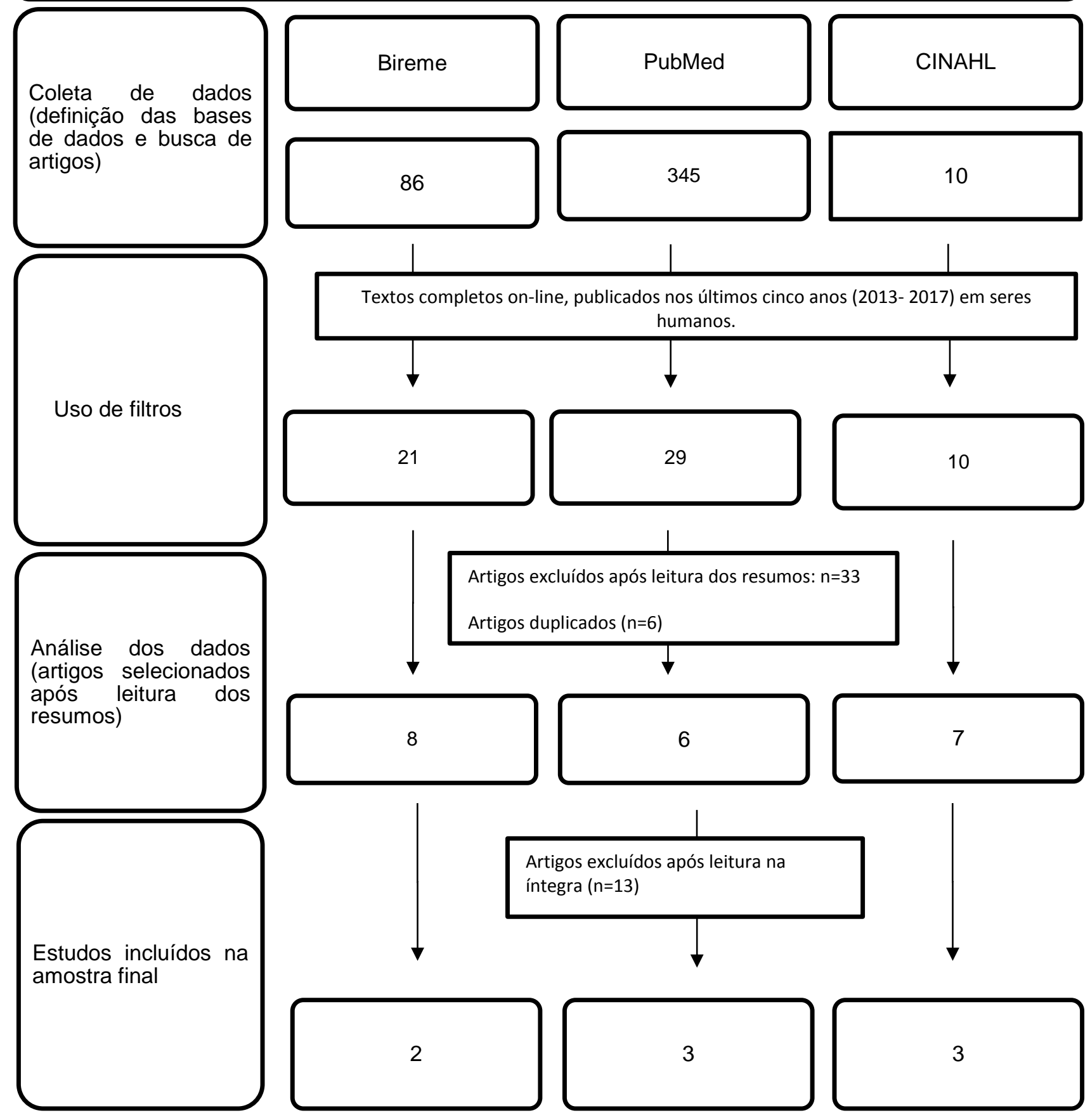

Fonte: Elaboração dos autores. 


\section{RESULTADOS}

Os oito estudos incluídos nesta revisão estavam no idioma inglês (100\%). A maioria das publicações foram concentradas no ano de 2017 (2/25\%), as demais publicações foram nos anos de 2008(1/12,5\%), 2009(1/12,5\%), 2013(1/12,5\%), 2014(1/12,5\%), 2015(1/12,5\%), e 2016(1/12,5\%) e não houve predomínio de estudos realizados no Brasil. Em relação à natureza do estudo, houve prevalência de estudos epidemiológicos $(08 / 100 \%)$. A principal linha de pesquisa investigada nessa temática versou sobre as alterações da autoestima em pacientes oncológicos, que estavam submetidos à quimioterapia (Quadro 3).

Os estudos avaliaram as alterações na autoestima de pacientes oncológicos, durante o tratamento quimioterápico, e como essas alterações afetavam o bem-estar físico e psíquico desses pacientes (Quadro 4).

Os estudos abordaram as alterações na autoestima em pacientes oncológicos durante o tratamento quimioterápico. A maioria dos estudos teve como enfoque principal as alterações da autoestima decorrente dos efeitos adversos da quimioterapia (LEMIEUX; MAUNSELL; PROVENCHER, 2008); (CAN et al., 2013); (CHANG et al., 2014) e como essas alterações afetam o bem estar do paciente (ROHDE et al., 2017); (PINQUART; FROHLICH, 2009); (BACKMAN et al., 2016); (CHANG et al., 2014) no convívio social (SHERIEF et al., 2015), na sua qualidade de vida e enfrentamento da doença.

\section{Alterações na autoestima do paciente oncológico}

A autoestima pode ser definida como o afeto positivo que o indivíduo tem de si próprio, sendo de grande importância na sua relação com os outros. Pacientes com câncer tendem a passar por várias alterações quanto a autoestima, desde a aceitação do diagnóstico e adesão aos tratamentos o que pode trazer impactos negativos quanto a sua autoimagem e bem estar (ROHDE et al., 2017); (CHEN et al,. 2018); (SHERIEF et aL, 2015).

É necessário que os profissionais de saúde percebam tais alterações e possam realizar medidas de apoio juntamente com esse paciente, quanto a orientações, apoio emocional, e atividades que possam está ajudando esses pacientes a lidarem melhor com a doença, promovendo o bem estar físico e emocional (PINQUART, FROHLICH, 2009); (BACKMAN et al, 2016); (SHERIEF et aL, 2015).

\section{Efeitos da quimioterapia na autoimagem}

Evidências mostraram que em pacientes oncológicos submetidos a quimioterapia apresentam baixo nível de autoestima em $84,83 \%$ dos pacientes, e em decorrência seus familiares apresentaram um nível de estresse psicológico significativo. Observou-se que quimioterapia possui um impacto no estado psicológico dos pacientes independente da faixa etária, pois crianças em tratamento quimioterápico, apresentaram alta prevalência de baixa autoestima e consequentemente um alto grau de estresse foram apresentados pelos seus pais (SHERIEF et AL, 2015).

São muito frequentes os efeitos adversos e a gravidade dos mesmos que está relacionado à quimioterapia dentre eles o efeito colateral mais comum é a alopecia de, sua probabilidade está relacionado ao tipo de droga utilizada e sua programação de administração. A alopecia é frequentemente considerada um efeito colateral inevitável e transitório, mas que afeta diretamente na imagem corporal (LEMIEUX; MAUNSELL, PROVENCHER, 2008).

As alterações na autoestima podem ocasionar uma condição caracterizada como estado psicológico baixo definido pela ocorrência dos seguintes eventos: o aparecimento de pensamentos negativos (LEMIEUX, MAUNSELL, PROVENCHER, 2008), Baixo senso de competência (CHANG et al, 2014), alta restrição (RODHE et al.,2017), depressão (PINQUART; FROHLICH, 2009), má relação com o cônjuge e familiares (BACKMAN et al., 2016) e altas variáveis de isolamento social (RODHE et al, 2017). E dentre os eventos adversos que também podem afetar na autoimagem, destacam-se o alto grau de estresse, alguns sintomas como náuseas, vômitos, anorexia, debilidade física, que provocam sentimento de impotência e desesperança afetando diretamente na autoestima (CAN et al, 2013); (LEMIEUX, MAUNSELL, PROVENCHER, 2008)

REAS/EJCH | Vol. 11 (3) | e190 | DOI: https://doi.org/10.25248/reas.e190.2019 Página 6 de 13 


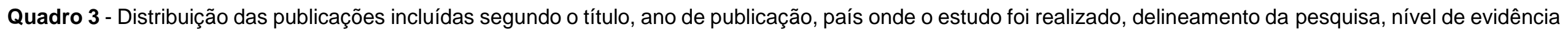
e grau de recomendação. Caxias, MA, 2018.

\begin{tabular}{|c|c|c|c|c|c|c|}
\hline Autor / Ano & Título & Base & País & Delineamento & NE & GR \\
\hline Lemieux J, et al. (2008) & $\begin{array}{l}\text { Chemotherapy-induced alopecia and effects on quality of life among women with } \\
\text { breast cancer: a literature review. }\end{array}$ & CINAHL & Canadá & $\begin{array}{l}\text { Revisão } \\
\text { Sistemática }\end{array}$ & 1 & $\mathrm{~B}$ \\
\hline Pinquart M, et al. (2009) & Psychosocial resources and subjective well-being of cancer patients. & CINAHL & Germânia & $\begin{array}{l}\text { Estudo de } \\
\text { Coorte }\end{array}$ & 3 & A \\
\hline Backman M, et al. (2016) & $\begin{array}{l}\text { Experiencing health - Physical activity during adjuvant chemotherapy treatment for } \\
\text { women with breast cancer. }\end{array}$ & Bireme & Suécia & $\begin{array}{l}\text { Estudo de } \\
\text { Coorte }\end{array}$ & 3 & A \\
\hline Can,Gulbeyaz, et al. (2013) & A comparison of men and women's experiences of chemotherapy-induced alopecia. & Bireme & Turquia & $\begin{array}{l}\text { Quanti- } \\
\text { qualitativo }\end{array}$ & 6 & A \\
\hline Rohde L, et al. (2017) & $\begin{array}{l}\text { Spiritual well-being in patients with the metastatic colorectal câncer receiving } \\
\text { Noncurative Chemotherapy: A qualitative study. }\end{array}$ & PubMed & Noruega & $\begin{array}{l}\text { Descritivo e } \\
\text { qualitativo }\end{array}$ & 6 & A \\
\hline Chen S, et al. (2017) & $\begin{array}{l}\text { Factors associated with healthcare professional's rating of disfigurement and self- } \\
\text { perceived body image in female patients with head and neck câncer. }\end{array}$ & CINAHL & Taiwan & $\begin{array}{l}\text { Descritivo e } \\
\text { qualitativo }\end{array}$ & 6 & A \\
\hline Chang O, et al. (2014) & $\begin{array}{l}\text { Association between socioeconomic status and altered appearance distress, body } \\
\text { image, and quality of life among breast cancer patients. }\end{array}$ & PubMed & EUA & $\begin{array}{l}\text { Descritivo e } \\
\text { qualitativo }\end{array}$ & 6 & $A$ \\
\hline Sherief LM, et al. (2015) & $\begin{array}{l}\text { Psychological Impact of Chemotherapy for Childhood Acute Lymphoblastic Leukemia } \\
\text { on Patientsand Their Parents. }\end{array}$ & PubMed & Egito & $\begin{array}{l}\text { Descritivo e } \\
\text { qualitativo }\end{array}$ & 6 & A \\
\hline
\end{tabular}

Fonte: Elaboração dos autores. NE = Nível de evidência, GR = Grau de recomendação.

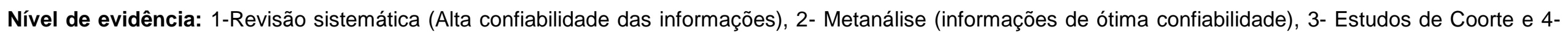

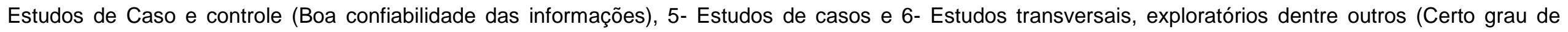

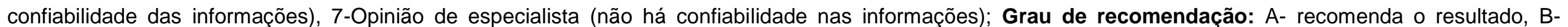
Resultados não conclusivos e ineficientes, C-Resultado é contraindicado.

REAS/EJCH | Vol. 11 (3) | e190 | DOI: https://doi.org/10.25248/reas.e190.2019 Página 7 de 13 
Quadro 4 - Publicações incluídas segundo objetivo principal, perfil amostral e principais resultados. Caxias, MA, 2018.

\begin{tabular}{|c|c|c|c|c|}
\hline Autor / Ano & Objetivo principal & Perfil amostral & Intervenções/Interesse & $\begin{array}{l}\text { Principais resultados } \\
\end{array}$ \\
\hline $\begin{array}{l}\text { Lemieux J, } \\
\text { et al. (2008) }\end{array}$ & $\begin{array}{l}\text { Descrever os efeitos } \\
\text { da alopecia na } \\
\text { qualidade de vida } \\
\text { (QV) em mulheres } \\
\text { com cancer de } \\
\text { mama. }\end{array}$ & $\begin{array}{lr}\text { Um total de } 38 \text { artigos } \\
\text { atenderam } r \text { aos } \\
\text { critérios resta } \\
\text { elegibilidade de desta } \\
\text { revisão e compuseram } \\
\text { a amostra final. }\end{array}$ & $\begin{array}{l}\text { Apontar as melhores evidências sobre os } \\
\text { efeitos da alopecia em vários aspectos da QV } \\
\text { em pacientes com câncer de mama, incluindo } \\
\text { ansiedade e sofrimento, imagem corporal, } \\
\text { sexualidade, autoestima, funcionamento social, } \\
\text { QV global e retorno ao trabalho. }\end{array}$ & $\begin{array}{l}\text { A perda de cabelo consistentemente está entre } \\
\text { os efeitos colaterais mais problemáticos da } \\
\text { quimioterapia, e foi descrita como angustiante, } \\
\text { e pode afetar a imagem corporal e a qualidade } \\
\text { de vida. }\end{array}$ \\
\hline $\begin{array}{l}\text { Pinquart M, } \\
\text { et al. (2009) }\end{array}$ & $\begin{array}{l}\text { Identificar as } \\
\text { mudanças nos } \\
\text { recursos psicológicos } \\
\text { de pacientes com } \\
\text { câncer durante a } \\
\text { quimioterapia. }\end{array}$ & $\begin{array}{l}150 \text { pacientes } \\
\text { recentemente } \\
\text { diagnosticados com } \\
\text { câncer em duas } \\
\text { unidades oncologicas. }\end{array}$ & $\begin{array}{l}\text { Avaliar as mudanças de recursos psicológicos, } \\
\text { e analisar o impacto dos recursos na adaptação } \\
\text { psicológica ao câncer. }\end{array}$ & $\begin{array}{l}\text { A aplicação dos recursos em pacientes } \\
\text { recentemente diagnosticados com cancer, } \\
\text { mostrou melhoria quanto ao bem estar } \\
\text { psicológico, facilitando tanto o entendimento } \\
\text { sobre o diagnostico do cancer, como no } \\
\text { enfrentamento aos tratamentos estressantes, } \\
\text { com efeitos a longo prazo. }\end{array}$ \\
\hline $\begin{array}{l}\text { Backman } \\
\text { M, et al. } \\
(2016)\end{array}$ & $\begin{array}{l}\text { Explorar como } \\
\text { mulheres com cancer } \\
\text { de mama } \\
\text { experimentam a } \\
\text { atividade física, } \\
\text { durante o tratamento } \\
\text { quimioterápico } \\
\text { adjuvante. }\end{array}$ & $\begin{array}{lr}16 & \text { mulheres } \\
\text { diagnosticas } & \text { com } \\
\text { cancer de mama. } & \end{array}$ & $\begin{array}{l}\text { Minimizar barreiras para adesão a atividade } \\
\text { física durante a doença e tratamento; capacitar } \\
\text { e motivar para se concentrar na saúde; } \\
\text { promover saúde e independência; }\end{array}$ & $\begin{array}{l}\text { A atividade física foi percebida como uma } \\
\text { ferramenta que apoiava os processos de saúde } \\
\text { e dava às mulheres uma sensação de alívio da } \\
\text { doença, tendo um impacto positivo na saúde, e } \\
\text { na melhora da autoestima. }\end{array}$ \\
\hline $\begin{array}{l}\text { Can,Gulbey } \\
\text { az, et al. } \\
\text { (2013) }\end{array}$ & $\begin{array}{l}\text { Determinar } \\
\text { incidência de } \\
\text { alopecia, relacionada } \\
\text { a quimioterapia e } \\
\text { como afeta a imagem } \\
\text { corporal e a } \\
\text { qualidade de vida dos } \\
\text { pacientes } \\
\text { cancer. }\end{array}$ & $\begin{array}{l}201 \text { homens e } 204 \\
\text { mulheres atenderam } \\
\text { aos critérios da } \\
\text { pesquisa. }\end{array}$ & $\begin{array}{l}\text { Avaliar e educar os pacientes através de } \\
\text { estratégias diferenciadas, para melhoria da } \\
\text { autoestima de pacientes que tiveram alopecia, } \\
\text { levando em consideração as características } \\
\text { culturais dos pacientes. }\end{array}$ & $\begin{array}{l}\text { A incidência de alopecia parcial ou completa foi } \\
\text { maior nas mulheres do que nos homens. } \\
\text { Consequentemente a imagem corporal e o } \\
\text { bem-estar psicológico dessas mulheres } \\
\text { encontravam-se inferiores do que comparado } \\
\text { nos homens. }\end{array}$ \\
\hline $\begin{array}{l}\text { Rohde L, et } \\
\text { al. (2017) }\end{array}$ & $\begin{array}{lr}\text { Avaliar o } & \text { bem-estar } \\
\text { espiritual, } & \text { em } \\
\text { pacientes } & \text { com } \\
\text { cancer de colorretal, } \\
\text { recebendo r a } \\
\text { quimioterapia na fase } \\
\text { paliativa. }\end{array}$ & $\begin{array}{l}20 \text { pacientes com } \\
\text { cancer de colorretal na } \\
\text { fase paliativa, com } \\
\text { idade entre } 34 \text { a } 75 \\
\text { anos. }\end{array}$ & $\begin{array}{l}\text { Utilizar estratégias para a harmonia interior, } \\
\text { compartilhar sentimentos, utilizar a fé como } \\
\text { suporte interno para lhe da com pensamentos } \\
\text { sobre o final da vida. }\end{array}$ & $\begin{array}{l}\text { Os profissionais de saúde precisam de } \\
\text { coragem e disposição suficiente para } \\
\text { compartilhar pensamentos, crenças, luto, afim } \\
\text { de orientar os pacientes para melhorar seu } \\
\text { bem-estar espiritual. }\end{array}$ \\
\hline
\end{tabular}

REAS/EJCH | Vol. 11 (3) | e190 | DOI: https://doi.org/10.25248/reas.e190.2019 Página 8 de 13 


\begin{tabular}{|c|c|c|c|c|}
\hline $\begin{array}{l}\text { Chen S, et } \\
\text { al. (2017) }\end{array}$ & $\begin{array}{lr}\text { Analisar os } & \text { fatores } \\
\text { como } & \text { efeitos } \\
\text { adversos } & \text { da } \\
\text { quimioterapia, } \\
\text { cirurgias, estágio e } \\
\text { localização r do } \\
\text { cancer, associados a } \\
\text { imagem corporal } \\
\text { autopercebida em } \\
\text { pacientes do sexo } \\
\text { feminino com câncer } \\
\text { de cabeça e pescoço. }\end{array}$ & $\begin{array}{l}105 \text { mulheres com } \\
\text { cancer de cabeça e } \\
\text { pescoço(CCP), em um } \\
\text { grande centro médico. }\end{array}$ & $\begin{array}{l}\text { Determinar os fatores associados a } \\
\text { classificação de desfiguração; Avaliar } \\
\text { cuidadosamente a aparência de mulheres com } \\
\text { o cancer; Ajudar os pacientes a lidar com a } \\
\text { desfiguração e obter uma melhor satisfação } \\
\text { com sua imagem corporal. }\end{array}$ & $\begin{array}{l}\text { Os Tratamentos médicos foram associados à } \\
\text { desfiguração de pacientes com CCP, causando } \\
\text { insastifacão à imagem corporal do paciente } \\
\text { auto percebida. O uso de intervenções de } \\
\text { camuflagem tais como o uso de cosméticos, } \\
\text { toucas, perucas podem ser usadas para ajudar } \\
\text { a lidar apropriadamente com a desfiguração e } \\
\text { para obter uma melhor satisfação com sua } \\
\text { imagem corporal. }\end{array}$ \\
\hline $\begin{array}{r}\text { Chang O, } \\
\text { et al. (2014) }\end{array}$ & $\begin{array}{l}\text { Estimar o impacto do } \\
\text { status } \\
\text { socioeconômico na } \\
\text { autoestima, angustia, } \\
\text { imagem corporal, e } \\
\text { qualidade de vida, } \\
\text { entre pacientes } \\
\text { coreanos com cancer } \\
\text { de mama. }\end{array}$ & $\begin{array}{l}126 \text { participantes com } \\
18 \text { anos ou mais, com } \\
\text { um diagnóstico câncer } \\
\text { de mama, que não } \\
\text { tinha evidência de } \\
\text { recidiva ou metástase, } \\
\text { e não tinha problemas } \\
\text { psicológicos no } \\
\text { momento da pesquisa. }\end{array}$ & $\begin{array}{l}\text { Proporcionar aos prestadores de cuidados } \\
\text { meios e estratégias para identificar as } \\
\text { mudanças e angústias em pacientes com } \\
\text { cancer de mama; fornecer informações } \\
\text { específicas e apoio psicossocial } \\
\text { a pacientes socioeconomicamenter mais } \\
\text { vulneráveis. }\end{array}$ & $\begin{array}{l}\text { As alterações na aparência ou imagem } \\
\text { corporal provocam problemas psicossociais, } \\
\text { físicos e funcionais, O r estado } \\
\text { socioeconômica também esta associada } \\
\text { com alterações da aparência } \\
\text { qualidade de vida. Foram observados que } \\
\text { os pacientes que tinham um emprego fixo, e } \\
\text { que estavam durante o tratamento } \\
\text { quimioterápico tinham pior imagem corporal do } \\
\text { que os pacientes desempregados. }\end{array}$ \\
\hline $\begin{array}{l}\text { Sherief LM, } \\
\text { et al. (2015) }\end{array}$ & $\begin{array}{l}\text { Avaliar a autoestima } \\
\text { de pacientes } \\
\text { pediátricos em } \\
\text { tratamento } \\
\text { quimioterápico para a } \\
\text { leucemia linfoblática } \\
\text { aguda, e o estado } \\
\text { psicológico de seus } \\
\text { pais. }\end{array}$ & $\begin{array}{l}178 \text { crianças que } \\
\text { estavam } \mathrm{em} \\
\text { tratamento } \\
\text { quimioterápico para } \\
\text { LLA e seus pais foram } \\
\text { avaliados quanto ao } \\
\text { índice de estresse. }\end{array}$ & $\begin{array}{l}\text { Utilizar uma escala de autoestima para } \\
\text { determinar o estado psicológico das crianças, e } \\
\text { o grau de estresse dos seus pais, através de } \\
\text { questionários, e de um sistema de classificação } \\
\text { e interpretação dos dados; Determinar um } \\
\text { programa de apoio psicossocial integrado e } \\
\text { multidisciplinar para os pacientes e seus pais. }\end{array}$ & $\begin{array}{l}\text { A quimioterapia para LLA tem um impacto } \\
\text { significativo no estado psicológico tanto dos } \\
\text { pacientes quanto de seus pais, com alta } \\
\text { prevalência de baixa autoestima em pacientes, } \\
\text { e alto grau de estresse psicológico em seus } \\
\text { pais. }\end{array}$ \\
\hline
\end{tabular}

Fonte: Elaboração dos autores. 


\section{Principais consequências da quimioterapia no cotidiano do paciente}

A quimioterapia está relacionada a diversas alterações na autoestima dos pacientes, decorrentes não só dos efeitos físicos, mas também psicológicos, tais alterações manifestam-se no cotidiano dos pacientes, prejudicando seu convívio social e aparência (BACKMAN et al, 2016); (LEMIEUX; MAUNSELL; PROVENCHER, 2008); (PINQUART; FROHLICH, 2009). .

A perda ou desfiguramento de alguma parte ou região do corpo, causa impactos imensuráveis quanto a aceitação da autoimagem e enfrentamento da doença (CHANG et al, 2014); (CHENG et al, 2018). E a alopecia que foi considerada um efeito colateral angustiante, causando conflitos na imagem corporal, pois como o cabelo é parte integrante da identidade humana, a perda de cabelo pode ter negativas repercussões em vários aspectos da qualidade davida (QV) (LEMIEUX, MAUNSELL, PROVENCHER, 2008).

Vários estudos indicam que as alterações na autoestima afetam diretamente no status socioeconômico e no retorno e no desempenho no trabalho, pois quando continuam a trabalhar ou retornam ao trabalho após o tratamento (CHANG et al, 2014), tendem a lidar com mudanças funcionais, apoio de colegas de trabalho ou olhares críticos dos mesmos (CHENG et al., 2018), flexibilidade de emprego, segurança e autoconfiaça em si mesmo, e em suas habilidades no trabalho (CHANG et al, 2014); (CHENG et al, 2018).

Essas mudanças diminuem o bem estar espiritual dos pacientes, e consequentemente a baixa Qualidade de Vida, afetando principalmente nos relacionamentos, por exemplo, as relações com o eu e os outros e a relação entre espiritualidade e religião. Por conta disse devem ser estabelecidas estratégias benéficas de enfrentamento espiritual que podem contribuir para um ajuste favorável à doença (LEMIEUX, MAUNSELL, PROVENCHER, 2008); (RODHE et al, 2017); (CHENG et al, 2018).

\section{Bundles de Intervenções}

Elencou-se um conjunto de cinco intervenções de enfermagem necessárias para orientar o paciente oncológico a compreender as mudanças na autoestima, e auxiliar na adaptação a essas alterações decorrentes da quimioterapia.

Quadro 5 - Bundles de Intervenções para orientação quanto as alterações na autoestima em pacientes oncológicos, submetidos a quimioterapia. Caxias-MA, 2018.

\begin{tabular}{|l|c|}
\hline \multicolumn{1}{|c|}{ INTERVENÇÕES } & $\begin{array}{c}\text { NÍVEL DE } \\
\text { EVIDÊNCIA }\end{array}$ \\
\hline $\begin{array}{l}\text { Estimular a prática de atividade física e outras atividades de lazer em pacientes } \\
\text { oncológicos. }\end{array}$ & III \\
\hline $\begin{array}{l}\text { Oferecer um programa de apoio psicossocial integrado para os pacientes e seus } \\
\text { acompanhantes no enfrentamento da doença e mudança na autoestima. }\end{array}$ & III \\
\hline $\begin{array}{l}\text { Orientar sobre o surgimento da alopecia durante a quimioterapia, e os efeitos } \\
\text { negativos na autoimagem. }\end{array}$ & VI \\
\hline $\begin{array}{l}\text { Promover atividades de apoio ao retorno ao trabalho e aconselhamento ocupacional } \\
\text { em pacientes oncológicos. }\end{array}$ & $\mathrm{VI}$ \\
\hline $\begin{array}{l}\text { Desenvolver atividades de embelezamento e do uso de adornos em pacientes } \\
\text { submetidos a quimioterapia. }\end{array}$ & $\mathrm{VI}$ \\
\hline
\end{tabular}

Fonte: Elaboração dos autores 


\section{DISCUSSÃO}

Os pacientes oncológicos sofrem diversos efeitos decorrentes da quimioterapia, efeitos estes que afetam significantemente a percepção do indivíduo quanto a sua imagem, em decorrência dessas alterações se faz necessário estabelecer condutas, que auxiliem o paciente a lidar com o tratamento e seus efeitos, minimizando os impactos na sua autoestima e bem estar.

Assim ao analisar os resultados dos estudos, os pesquisadores escolheram cinco intervenções para compor o bundle. Onde, o bundle constitui-se pelas seguintes intervenções e níveis de evidência III e VI: Estimular a pratica de atividade física e outras atividades de lazer em pacientes oncológicos; Oferecer um programa de apoio psicossocial integrado para os pacientes e seus acompanhantes no enfrentamento da doença e mudança na autoestima; Fornecer informações específicas apoio psicológico a pacientes e família; Orientar sobre o surgimento da alopecia durante a quimioterapia, e o efeitos negativos na autoimagem; Desenvolver atividades de embelezamento e do uso de adornos em pacientes submetidos a quimioterapia.

\section{Estimular a prática de atividade física e outras atividades de lazer em pacientes oncológicos}

O baixo nível de atividade física em pacientes oncologicos está assosicado ao tratamento quimioterápico e seus efeitos adversos, tais como a carga de sintomas, e os ligados a aparência corporal, como por exemplo, cicatrizes cirúrgicas, e a perda de cabelo, são os mais frequentes a não adesão de atividade fisica durante 0 tratamento (BACKMAN et al, 2016).

A prática de atividade física durante o tratamento quimioterápico mostrou-se um componente importante para o tratamento da doença, apesar dos efeitos adversos decorrente do tratamento, a atividade física revelou-se como uma medida eficaz para alívios dos sintomas, e melhora no bem estar físico (BACKMAN et al, 2016). Sendo vista como uma ferramenta para manter e recuperar a saúde física, mental e social, propocionando envolvimento e responsabilidade pelo próprio cuidado e pela própria saúde (BACKMAN et al, 2016).

\section{Oferecer um programa de apoio psicossocial integrado para os pacientes e seus acompanhantes no enfrentamento da doença e mudança na autoestima}

O diagnostico de câncer representa uma importante ameaça ao bem-estar subjetivo, ocasionando reações emocionais nos individuos que afentem na compreensão do diagnostico e na realização terapêutica (PINQUART, FROHLICH, 2009). Os principais estressores para os pacientes e seus acompanhantes estão relacionados ao tratamento, perda de controle, ambiente hospitalar, recidivas, o medo de morrer, má imagem corporal, falta de auto-estima e dificuldades de transição na vida social (SHERIEF et al, 2015).

O apoio psicossocial,pode representar uma fonte importante no tratamento do câncer, promovendo relações positivas nos papéis pessoais, bem estar e autoestima, que protege a saúde psicológica, diminuindo os efeitos dos estressores relacionados ao câncer (PINQUART, FROHLICH, 2009). Esse apoio psicossocial deve ser desenvolvido náo somente com o paciente oncologico, mas tambem com o seus acompanhantes, que direcionam toda a atenção apenas para o doente e seu acompanhamento integral no tratamento, levando a mudanças na rotina da vida diária, comprometimento do autocuidado, e conflitos com o conjuge ou demais familiares (SHERIEF et al, 2015). Que podem ocasionar distúrbios do sono e alterações no humor PINQUART, FROHLICH, 2009).

Um programa de apoio psicossocial integrado para os pacientes e seus acompanhantes é essencial para ajudá-los a lidar com os estresses que enfrentam, responder suas perguntas, ouvi-los pacientemente , ajudálos a expressar suas emoções, dar explicações, conselhos e apoio (SHERIEF et al, 2015).

\section{Orientar sobre o surgimento da alopecia durante a quimioterapia, e os efeitos negativos na autoimagem.}

A incidência de alopecia é estimada em $65 \%$ nos tratamentos quimioterápicos, e é considerada um dos fatores mais temidos e traumáticos no tratamento de pacientes com câncer (CAN et al, 2013). A alopecia está associada a uma perda de privacidade, porque isso torna o ambiente consciente de que a pessoa está recebendo a quimioterapia, e é também um visível lembrete da doença (LEMIEUX, MAUNSELL; PROVENCHER, 2008). 
A natureza visual da alopecia pode afetar a imagem corporal, a qualidade de vida, a interação social e a sexualidade do paciente. Levando ao sofrimento emocional, problemas pessoais, sociais e relacionados ao trabalho (CAN et al, 2013). Por conta do sentimento de estigmatização, e das alterações na identidade do indivíduo de uma pessoa saudável para a de um paciente com câncer (LEMIEUX; MAUNSELL; PROVENCHER, 2008). É necessário orientar quanto ao efeito da alopecia, percepções da perda de cabelo, Qualidade de Vida, angústia psicológica, ansiedade, imagem corporal que podem afetar o paciente, provocando alterações na autoestima (CAN et al, 2013); (LEMIEUX, MAUNSELL; PROVENCHER, 2008).

\section{Promover atividades de apoio ao retorno ao trabalho e aconselhamento ocupacional em pacientes oncológicos}

O sofrimento ocasionado pelos efeitos adversos da quimioterapia resulta em mudanças que afetem nas mais diversas esferas da vida do paciente, e em diferentes níveis socioeconômicos (CHENG et al, 2018); (LEMIEUX, MAUNSELL, PROVENCHER, 2008). O retorno ao trabalho pode significar em decorrência dos diferentes níveis de alteração na aparência, aflição, agústia e baixo rendimento no trabalho para o paciente (CHANG et al, 2014), e que podem se agravar com a falta de recursos (PINQUART, FROHLICH, 2009), como informação, falta de apoio social, o que pode dificultar sua capacidade para enfrentar as barreiras gerenciar o sofrimento (CHANG et al, 2014).

Os profissionais de saúde devem estar atentos para as mudanças e aflições que os pacientes com câncer (CHANG et al, 2014); (CHENG et al, 2018); (LEMIEUX; MAUNSELL, PROVENCHER, 2008). Com isso, é necessário fornecer informações específicas, realizar intervenções e ofercer suporte psicossocial para pacientes socioeconomicamente vulneráveis, e voltados para o retorno e desempenho no trabalho (CHANG et al, 2014), para que os pacientes consigam lidar com as mudanças físicas e as aflições emocionais e prosseguir suas atividades diárias e de trabalho (CHANG et al, 2014); (CHENG et al, 2018).

\section{Desenvolver atividades de embelezamento e do uso de adornos em pacientes submetidos a quimioterapia.}

Pacientes submetidos a quimioterapia sentem-se mais ansiosos (CHANG et al, 2014), com relação as mudanças na aparência, se sentem menos seguros com relação si mesmo e em publico, pois acham que os pessoas estão the observando, vendo as altreações no rosto e no corpo decorrente do tratamento (CHANG et al, 2014); (CHENG et al, 2018); (PINQUART, FROHLICH, 2009), tentam esconder a alopecia (CHANG et al., 2014), pois todas essas alterações são vistas como estigma negativo em relação ao paciente com cancer (CHANG et al, 2018); (LEMIEUX, MAUNSELL, PROVENCHER, 2008).

Em decorrência as insatisfação com a imagem corporal em pacientes oncologicos, é nessário que os profissionais de enfermagem avaliem cuidadosamente as alterações na aparência dos pacientes, e utilizar estratégias que visem melhorar a satisfação com a imagem corporal, tais como Intervenções de camuflagem, como o uso de perucas, técnicas em embelezamento, como por exemplo, o uso de cosmeticos (CHANG et al, 2018), e a técnica de resfriamento capilar (LEMIEUX, MAUNSELL, PROVENCHER, 2008), que podem ser usadas para ajudar a lidar adequadamente com a desfiguração, alopecia, e outras alterações decorrentes da quimioterapia.

Os profissionais de saúde devem frequentemente realizar a avaliação do estado psicologico dos pacientes assim como observar o aparecimento de sinais e sintomas que possam indicar alterações na autoestima (LEMIEUX, MAUNSELL, PROVENCHER, 2008), através dos fatores de riscos relacionados a alterações na autoestima o profissional de saúde pode estar realizando atividades recreativas e dinâmicas, grupos de discussões e pequenas ações como elogios, incentivar o uso de adornos, maquiagem no dia a dia dos pacientes (BACKMAN et al, 2016);(CAN et al., 2013); (LEMIEUX, MAUNSELL, PROVENCHER, 2008), é importante que o profissional estabeleça um vinculo de segurança e confiabilidade com o seu cliente para que ele se sinta confortavel para conversar sobre a sua experiência com o tratamento, diagnotico, presença de pensamentos negativos ou sensação de tristeza e que também se sinta apto e confiante a reverter os sinais de alteração na autoestima (CHANG et al, 2014); (CHENG et al, 2018).

REAS/EJCH | Vol. 11 (3) | e190 | DOI: https://doi.org/10.25248/reas.e190.2019 Página 12 de 13 


\section{CONSIDERAÇÕES FINAIS}

O tratamento quimioterapico pode ser considerado um fator determinante nas alterações da autoestima, pois possibilita a ocorrência de inúmeros impactos na vida do paciente oncológico, tais como, fisíco, emocionais e sociais, que foram descritos detalhadamente no estudo. Dentre as alterações psicologicas destacou-se mudanças na auto imagem, que afeta a vida do paciente em vários aspectos, levando ao desenvolvimento de outros problemas como alterações de humor, isoloamento social, e depressão.

Os profissionais de saúde devem estar atentos ao aparecimento de sinais e sintomas que possam indicar alterações na autoestima, pois a detectação precoce que pode contibuir para intervir de forma significativa na melhora do quadro do paciente, facilitando sua aceitação frente ao enfrentamento da doença e as alterações apresentadas pela mesma.

As intervenções do bundles trazem medidas que versem sobre as ações assistenciais que envolvam a melhora na autoestima na vida dos pacientes em tratamento quimioterápico, bem como ações especificas e inidividualizadas que devem ser utilizadas pelos profissionais na aplicação da assistência de enfermagem.

\section{REFERÊNCIAS}

1. BACKMAN M et al. Experiencing health - Physical activity during adjuvant chemotherapy treatment for women with breast cancer. European journal of oncology nursing. April 2016, Volume 21, Pages 160-167.

2. BORDA MM, RINCÓN M.E, PEREZ Ma. A. impacto de la reconstrunccion mamária sobre la autoestima. Univ Psychol. 2012;11(1): 25-41.

3. BRASIL. Ministério da Saúde. O que é o câncer. Inca: Rio de Janeiro, 2009.

4. BRASIL. Ministério da Saúde. Secretaria de Atenção à Saúde. Protocolos clínicos e diretrizes terapêuticas em Oncologia/Ministério da Saúde, Secretaria de Atenção à Saúde - Brasília : Ministério da Saúde, 2014.

5. BROOME M.E. Integrative literature reviews for the development of concepts. In: RODGERS, B.L.; CASTRO, A.A. Revisão sistemática e meta-análise. 2006.

6. CAN G et al. A comparison of men and women's experiences of chemotherapy-induced alopecia. June 2013.Volume 17, Issue 3, Pages 255-260

7. $\mathrm{CHANG} O$ et al. Association between socioeconomic status and altered appearance distress, body image, and quality of life among breast cancer patients. Câncer Asiático Pac J Prev. 2014; 15 (20): 8607-12.

8. CHEN S et al. Factors associated with healthcare professional's rating of disfigurement and self-perceived body image in female patients with head and neck cance. Eur J Cancer Care. 2018;27:e12710.

9. FORONES M.N et al. A comunicação no cuidado ao paciente, uma questão fundamental na enfermagem. Rev. Brasileira de Enfermagem, Brasília, 2006.

10. LEMIEUX J, MAUNSELL E, PROVENCHER L. Chemotherapy-induced alopecia and effects on quality of life among women with breast cancer: a literature review. Psycho-Oncology 1: 317-328 (2008).

11. MARQUES P, PIERIN AMG. Fatores que influenciam a adesão de pacientes com câncer à terapia antineoplásica oral. Acta Paul Enferm 2008;21(2):323-9.

12. MINAYO M.C.S. O cliente submetido a quimioterapia oncológica, sob ótica compreensiva do enfermeiro. Rev.Enfermagem atual, 2002.

13. PINQUART M, FROHLICH C. Psychosocial resources and subjective well-being of cancer patients. Psychology and Health. Vol. 24, No. 4, April 2009, 407-421.

14. ROHDE L et al. Spiritual well-being in patients with the metastatic colorectal câncer receiving Noncurative Chemotherapy: A qualitative study. Cancer Nurs. 2017 May/Jun;40(3):209-216.

15. SCHMIDT AM, HAHN GV. Qualidade de vida de pacientes em tratamento quimioterápico. Revista destaques acadêmicos, vol. 6, n. 3, 2014 - ccbs/univates.

16. SHERIEF LM et al. Psychological Impact of Chemotherapy for Childhood Acute Lymphoblastic Leukemia on Patientsand Their Parents. Medicine (Baltimore). 2015 Dec; 94(51): e2280.

17. SILVA J, SILVA MJS. ABC do câncer: abordagens básicas para o controle do câncer. INCA-Instituto Nacional de Câncer;- 3. Ed. rev. atual. - Rio de Janeiro: Inca, 2017.

18. SMELTZER SC, BARE BG. Intervenção de enfermagem ao paciente oncológico com dor. Aq. Ciência saúde, 2005.

19. SOARES E M, SILVA S R. Perfil de pacientes com câncer em tratamento quimioterápico. Rev. Brasileira Enf. 2010; 63 (4): 51. 